City University of New York (CUNY)

CUNY Academic Works

1982

\title{
Exotic Levels from Topology in the Quantum-Chromodynamic Effective Lagrangian
}

\author{
A. P. Balachandran \\ Syracuse University \\ V. Parameswaran Nair \\ City College of New York \\ S. G. Rajeev \\ Syracuse University \\ A. Stern \\ University of Texas, Austin
}

\section{How does access to this work benefit you? Let us know!}

More information about this work at: https://academicworks.cuny.edu/cc_pubs/475

Discover additional works at: https://academicworks.cuny.edu

This work is made publicly available by the City University of New York (CUNY).

Contact: AcademicWorks@cuny.edu 
gestions and for criticism of the manuscript and Daniel S. Fisher for helpful discussions and for his hospitality at Bell Laboratories, where this work was begun. The support of the National Science Foundation is also acknowledged.

\footnotetext{
(a) Permanent address.

${ }^{1}$ R. J. Baxter, J. Phys. A 13, L61 (1980).

${ }^{2}$ R. J. Baxter, J. Stat. Phys. 26, 427 (1981).

${ }^{3}$ R. J. Baxter and P. A. Pearce, J. Phys. A $\underline{15}, 897$ (1982).

${ }^{4}$ M. P. M. den Nijs, J. Phys. A 12, 1857 (1979).

${ }^{5}$ B. Nienhuis, E. K. Riedel, and $\vec{M}$. Schick, J. Phys. A 13 , L189 (1980); R. B. Pearson, Phys. Rev. B $\underline{22}$, $2579(1980)$.

${ }^{6}$ B. Nienhuis, J. Phys. A 15, 199 (1982).

${ }^{7}$ S. Alexander, Phys. Lett. 54A, 353 (1975).

${ }^{8}$ E.g., E. Domany, M. Schick, J. S. Walker, and R. B. Griffiths, Phys. Rev. B 18, 2209 (1978).

${ }^{9}$ D. S. Gaunt and M. E. Fisher, J. Chem. Phys. 43 , 2840 (1965); R. J. Baxter, I. G. Enting, and S. K.
}

Tsang, J. Stat. Phys. 22, 465 (1980).

${ }^{10}$ See, e.g., M. E. Fisher, in Proceedings of the Twenty-Fourth Nobel Symposium on Collective Properties of Physical Systems, edited by Bengt Lundqvist and Stig Lundqvist (Academic, New York, 1973), p. 16, and in Magnetism and Magnetic Materials - 1974, edited by C. D. Graham, G. H. Lander, and J. J. Rhyne, A.I.P. Conference Proceedings No. 24 (American Institute of Physics, New York, 1975), p. 273.

${ }^{11}$ Baxter also calculates the sublattice order parameter in regime IV, finding $R \sim|p|^{1 / 4}$. This may be contrasted with the "prediction" of $R \sim|p|^{3 / 32}$ for the dilute Ising tricritical point, with use of the conjectured leading field exponent, $y_{H}=\frac{77}{40}$, of Ref. 5. The source of this discrepancy is at present unclear; one possible, if unlikely, explanation is that the order parameter is linked to a dangerous irrelevant variable. Such a dangerous irrelevant variable does play an important role near tricriticality for dimensionalities $d \geqslant 3$; see

S. Sarbach and M. E. Fisher, Phys. Rev. B 18,2350 (1978).

${ }^{12}$ A. Aharony and M. E. Fisher, Phys. Rev. Lett. $\underline{45}$, $679(1980)$.

\title{
Exotic Levels from Topology in the Quantum-Chromodynamic Effective Lagrangian
}

\author{
A. P. Balachandran, V. P. Nair, and S. G. Rajeev \\ Physics Department, Syracuse University, Syracuse, New York 13210 \\ and \\ A. Stern \\ Center for Particle Theory, University of Texas, Austin, Texas 78712 \\ (Received 23 April 1982)
}

\begin{abstract}
Skyrme has shown that the $\mathrm{SU} \otimes \mathrm{SU}(2)$ chiral model has nontrivial topological sectors with static solutions for suitable Lagrangians. The baryon number $B$ and strangeness of these sectors have been studied, and the existence of bound states of the nucleon field to the lightest solitons is shown. It is found that there must be long-lived levels with $|B| \gtrless 6$ and $|s| \gtrsim 6$ and $1.8 \mathrm{GeV} \lesssim m \lesssim 5.6 \mathrm{GeV}$, some having half-integral charge and exotic relation between $B$ and $s$, that can be pair produced in, say, $e^{+} e^{-}$collisions.

PACS numbers: $12.35 . \mathrm{Eq}, 11.30 . \mathrm{Rd}, 14.80 . \mathrm{Pb}$
\end{abstract}

It is well known that the low-energy behavior of QCD, i.e., pion-nucleon physics, can be well described by the chiral SU(2) $)_{L} \mathrm{SU}(2)_{R}$ effective Lagrangian, ${ }^{1}$

$$
\mathscr{L}=\frac{1}{2} f_{\pi}^{2} \operatorname{Tr}\left(\partial_{\mu} u^{\dagger} \partial_{\mu} u\right)+\left(32 e^{2}\right)^{-1} \operatorname{Tr}\left\{\left[\partial_{\mu} u u^{\dagger}, \partial_{\nu} u u^{\dagger}\right]^{2}\right\}+\ldots=\mathscr{L}_{0}+\mathscr{L}_{1}+\ldots,
$$

where $f_{\pi}=67 \mathrm{MeV} . u(x)$ is a $2 \times 2 \mathrm{SU}(2)$ matrix. For all finite-energy configurations, $u(x) \rightarrow 1$ as $|\overrightarrow{\mathrm{x}}| \rightarrow \infty$.

The term $\mathscr{L}_{0}$ gives the standard current-algebra results. Terms quartic in the derivatives, like $\mathcal{L}_{1}$, appear when we include results beyond the soft-pion limit or from renormalization effects. ${ }^{2}$

In this Letter, we discuss some unusual conse- quences of the Lagrangian (1) associated with soliton solutions. Skyrme $e^{3,4}$ has shown that this model has nontrivial topological sectors labeled by the integer-valued charge

$$
t=\left(48 \pi^{2}\right)^{-1} \epsilon_{i j k} \int d^{3} x \operatorname{Tr}\left(I_{i}\left[I_{j}, I_{k}\right]\right),
$$

where $I_{i}=\partial_{i} u u^{\dagger}$. If $e \neq \infty$, the sectors $t \neq 0$ admit 
static solutions of the form,,$^{3,5-7}$

$$
u(\vec{x})=\cos \theta(r)+i \vec{\tau} \cdot \hat{x} \sin \theta(r),
$$

where

$$
t=\pi^{-1}[\theta(0)-\theta(\infty)] .
$$

For the Ansatz

$$
\cos \theta(r)=\frac{1-(r / R)^{2}}{1+(r / R)^{2}}, \quad \sin \theta(r)=\frac{2(r / R)}{1+(r / R)^{2}}
$$

with variational parameter $R$, the minimum of energy for $\mathscr{L}_{0}+\mathscr{L}_{1}$ occurs when $R=R_{0} \simeq 1 / 2 e f_{\pi}$. This gives an estimate for the soliton mass $M$,

$$
M=E_{\text {static }} \simeq 118 f_{\pi} / e \text {. }
$$

We shall estimate the numerical magnitude of $e$ (and hence $M$ ) later. The value of $M$ is not sensitive to the form of the Ansatz for $\theta(r)$.

Our investigation is aimed at understanding the properties of these solitons, particularly their quantum numbers, by studying the bound states of fermions coupled to these. We follow the strategy of Goldstone and Wilczek ${ }^{8}$ and Jackiw and co-workers. ${ }^{9}$ Nucleons couple to the pions through chirally invariant Yukawa terms of the form

$$
\begin{aligned}
& \mathcal{L}^{\prime}=-m\left(\bar{N}_{R} u N_{L}+\text { H.c. }\right)=-m \bar{N} U N, \\
& U=\cos \theta(r) \underline{1}+i \vec{\tau} \cdot \hat{x} \gamma_{5} \sin \theta(r) .
\end{aligned}
$$

where 1 is the unit matrix.

Let $|t\rangle$ denote the ground state of the solitonic sector with topological number $t$. The notation suppresses a possible degeneracy index..$^{3-5}$ On the basis of known results and techniques, ${ }^{8,9}$ for couplings of the form (7), it can be shown that the baryon number $B$ of $|t\rangle$ is $t$, i.e.,

$$
\left\langle t\left|\int d^{3} x N^{\dagger} N\right| t\right\rangle=t\langle t \mid t\rangle \text {. }
$$

For a baryon $N^{(I)}$ of isospin $I$, the coupling analogous to (7) is

$$
\mathcal{L}_{I}{ }^{\prime}=-m_{I}\left(\bar{N}_{R}^{(I)} u_{I} N_{L}{ }^{(I)}+\text { H.c. }\right),
$$

where $u_{I}$ is the representative of $u$ in the representation with isospin $I$. For this interaction, we have

$$
\left\langle t\left|\int d^{3} x N_{I}^{\dagger} N_{I}\right| t\right\rangle=\left(48 \pi^{2}\right)^{-1} \epsilon_{i j k} \int d^{3} x \operatorname{Tr}\left(I_{i}^{(I)}\left[I_{j}^{(I)}, I_{k}^{(I)}\right]\right)=\frac{I(I+1)(2 I+1)}{\frac{1}{2}\left(\frac{1}{2}+1\right) 2} t .
$$

[Notice that $\operatorname{Tr} I_{i}\left[I_{j}, I_{k}\right] \epsilon_{i j k}$ is proportional to the trace of the quadratic Casimir operator for the representation $I$, i.e., $\sim I(I+1)(2 I+1)$. Equation (10) is the properly normalized version of this statement.]

Thus for the baryon octet of the eightfold way (with nucleon and $\Xi$ doublets and $\Sigma$ triplet), we have $B=6 t$ for the ground state $|t\rangle$. The coupling of additional fermions will increase this number. (We note here that it has been suggested before that $B= \pm t_{0}^{3,5}$ ) A similar calculations shows that the strangeness of $|t\rangle$ is $-6 t$ in the presence of the baryon octet. [The relation $Y=0$ is not, however, maintained for an arbitrary SU(3) multiplet of baryons.] Thus we reach our first conclusion: These solitons are characterized by relatively high baryon number and strangeness $>6$ in magnitude).

An unambiguous theoretical determination of spin $s$ and isospin $I$ of these states is known to be difficult. Skyrme and other $\mathrm{s}^{1-3}$ have argued that the states $| \pm 1\rangle$ have $s=I=\frac{1}{2}$. In any event, depending on the value of $Y$ and $I$, such states may be fractionally charged (if, for instance, the electric charge is given by the usual formula $Q=I_{3}$ $+Y / 2$ ). They may also have an exotic connection between $B$ and $s$ (for instance, even $B$ and halfinteger $s$ ).
The bound states of the nucleon $N$ to the soliton $u$ are determined by the Hamiltonian

$$
H=\vec{\alpha} \cdot \overrightarrow{\mathrm{p}}+m \beta U .
$$

This form of the Hamiltonian is awkward for approximate computations of the spectrum because of the large factor $m$ in the interaction. To circumvent this difficulty, we first make the unitary transformation

$$
\begin{aligned}
& H^{\prime}=V H V^{\dagger}=\vec{\alpha} \cdot \overrightarrow{\mathrm{P}}+\beta m, \quad \overrightarrow{\mathrm{P}}=\overrightarrow{\mathrm{p}}-i u \nabla u^{\dagger}\left(1+\gamma_{5}\right) / 2, \\
& V=u\left(1+\gamma_{5}\right) / 2+\underline{1}\left(1-\gamma_{5}\right) / 2 .
\end{aligned}
$$

If the soliton size $R_{0}$ is large compared to $\mathrm{m}^{-1}$, $H^{\prime}$ can be simplified by standard Foldy-Wouthuysen transformations. ${ }^{10}$ The new Hamiltonian up to leading terms in $1 / \mathrm{m}$ is

$$
\begin{aligned}
& H^{\prime \prime}=\beta\left|(2 m)^{-1}(\vec{\alpha} \cdot \vec{\beta})^{2}+m\right|-i \vec{\alpha} \cdot u \nabla u^{\dagger} \gamma_{5} / 2, \\
& \vec{\beta}=\overrightarrow{\mathrm{p}}-i u \nabla u^{\dagger} .
\end{aligned}
$$

It commutes with $\vec{J}=\overrightarrow{\mathrm{r}} \times \overrightarrow{\mathrm{p}}+\vec{\sigma} / 2+\vec{\tau} / 2$ [where the Pauli matrices $\vec{\sigma}$ and $\vec{\tau}$ act on spin and isospin labels, respectively, if $u$ has the form (3)].

The Hamiltonian $H^{\prime \prime}$ has no zero-energy bound state in contrast to many previous examples..$^{8,9}$ 
But it does have (at least) one positive-energy bound state with $\vec{J}=0$ if $u$ has $t=-1$ [and a negative-energy bound state with $\vec{J}=0$ if $u$ has $t=+1$ ] if $e$ is not too large. The differential equation for the $\vec{J}=0$ bound-state wave function can be found by standard methods. ${ }^{9}$ The details will be given elsewhere. In the $\beta$-diagonal representation, the last two components of positive-energy solutions are zero while the first two components can be written as $\varphi_{a \alpha}$ where $a$ and $\alpha$ are isospin and spin labels. We can use the Ansatz

$$
\Phi_{a \alpha}=c \epsilon_{a \alpha} e^{-k r}, \quad \tilde{\epsilon}=-\epsilon, \epsilon_{12}=+1
$$

(where the variational parameter $1 / k$ is a measure of the size of the bound state and $c$ is the normalization constant) and the form (7) for $\theta$ to estimate the bound-state energy in this representation. The eigenvalue $\mu$ of $H^{\prime \prime}$ found in this way is

$$
\mu \simeq m-3 \cdot 4 f_{\pi} e .
$$

It occurs when $1 / k$ equals $1 / k_{0} \simeq\left(0.17 / f_{\pi}\right)(1 / e)$. (The value of $\mu$ does not sensitively depend on the form of $\varphi$.) If the soliton energy is $M$, the actual mass of this bound state including the background soliton energy is $M+\mu$.

If $|-1\rangle$ denotes the $t=-1$ soliton state (with filled Dirac sea and mass $M$ ), then the nucleon bound to the soliton has the state $a^{\dagger}|-1\rangle$ where $a^{\dagger}$ is the bound-state creation operator. It is defined by second quantizing $N$ (Ref. 9):

$$
\begin{aligned}
& N=a \Psi+\text { continuum terms, } \\
& \left\{N_{\alpha}(\overrightarrow{\mathrm{x}}, t), N_{\beta}^{\dagger}{ }^{\dagger}(\overrightarrow{\mathrm{y}}, t)\right\}=\delta_{\alpha \beta} \delta^{3}(x-y) .
\end{aligned}
$$

Here $\Psi$ is the bound-state wave function. The state $a^{\dagger}|-1\rangle$ is nondegenerate if $|-1\rangle$ has $I=s=0$. If not, its spin, isospin, and degeneracy are given by that of $|-1\rangle$. Note that there is only one creation operator $a^{\dagger}$ because there is only one bound state with $\overrightarrow{\mathrm{J}}=0$ to the field $u$. Therefore $a^{\dagger}$ carries zero $s$ and $I$.

The baryon number $B$ of $a^{\dagger}$ is 1 while, as we saw, it has $I=s=0$. Thus it is exotic, with fractional $\left(+\frac{1}{2}\right.$ ) electric charge $Q=I_{3}+Y / 2$ and "wrong" relation between $B$ and $s$ (being bosonic in spin, but with odd $B) .{ }^{11}$ The consequence is striking: Either the state $|-1\rangle$ or the state $a^{\dagger}|-1\rangle$ is exotic, having half-integral $Q$ and "wrong" relation between $B$ and $s$. (We suppose here that either $|-1\rangle$ or $a^{\dagger}|-1\rangle$ is a normal state.)

Analogous considerations apply to the sector $t=+1$, and to the bound states of other baryons $(\Sigma, \Xi, \ldots)$. There are also rotational excitations in the soliton sectors $\mathrm{s}^{3,5}$ and their partners created by $a^{\dagger}$.
The possibility of exotic states in the presence of solitons is very well known in the literature. ${ }^{8,9}$ What is interesting is that such states seem to occur in the chiral model which is so close to present-day experiments in particle physics.

A numerical estimate of the soliton mass $M$ can be obtained as follows. Note that $e$ cannot be too small and hence $M$ cannot be too large if $\mathcal{L}$ is dominated by the first two terms since a small value of $e$ leads to a large contribution to lowenergy $\pi-\pi$ scattering. Now $\mathcal{L}_{1}$ does not affect $S-$ wave scattering lengths while its contribution to the $P$-wave scattering length $a_{1}{ }^{1}$ is $\left(1 / 32 \pi e^{2}\right)\left[m_{\pi} /\right.$ $\left.f_{\pi}\right]^{4}$. This is a correction to the standard currentalgebra value 0.03.12 Experimentally two conflicting numbers $\simeq 0.04$ and 0.1 have been reported for $a_{1}{ }^{1}$. This suggests the estimate

$$
0.07 \gtrsim\left(32 \pi e^{2}\right)^{-1}\left(m_{\pi} / f_{\pi}\right)^{4} \gtrsim 0.01,
$$

or for $m_{\pi} \simeq 137 \mathrm{MeV}, 2.48 \lesssim e^{2} \lesssim 17.39$. The values of $M$ and $\mu$ can now be estimated from (6) and (15), $1.9 \mathrm{GeV} \lesssim M \lessgtr 5 \mathrm{GeV}$ and $-12 \mathrm{MeV} \lesssim \mu_{\omega} \lesssim 579$ $\mathrm{MeV}$. We also find $0.25 m_{\pi}{ }^{-1} \lesssim R_{0} \lesssim 0.65 m_{\pi}{ }^{-1}$ and $0.09 m_{\pi}^{-1} \lesssim 1 / k_{0} \lesssim 0.22 m_{\pi}^{-1}$. For $e^{2}$ as large as $17.39,1 / R_{0} m$ is of order of 0.6 and is large while our approximation methods for $\mu$ are valid only if it is small. The lower limit for $\mu$ is thus unreliable.

The $t \neq 0$ levels are characterized by large $B$ and strangeness. The production of these levels by decay of normal matter is thus suppressed. The combination of these properties with low mass also makes the $|t|=1$ levels very stable. The pair production of these states, which are extended, is likely to be inhibited by form-factor effects. Thus such states do not seem to be ruled out by available data. We shall now argue that there is a further suppression factor, at least as small as about $10^{-4}$, for the rate of any $t$-nonconserving process (for instance, the annihilation of these states with normal matter with normal final states) which makes such states even harder to see.

We recall that Skyrme's topological charge is not conserved in the $\sigma$ model if the $\sigma$ field is allowed to fluctuate to zero. Thus if we formulate the latter model in terms of, say, a $2 \times 2$ matrix $M$ with $M^{\dagger} M=\sigma^{2}$, then so long as $\sigma \neq 0$, we can define our $u$ as $M / \sigma$ without encountering singularities, and $t$ is conserved. But if $\sigma$ develops zeros, $u$ is undefined at these zeros and $t$ need not be conserved. A semiclassical estimate of the suppression factor induced by such nonconservation of $t$ is provided by saturating the func- 
tional integral by the two-meron solution ${ }^{13}$ adapted to chiral models ${ }^{14}$; this solution can change $t$ by 1 . The positions of the singularities of the solution are interpreted as idealizations of the zeros of $\sigma$. If $A$ is the action for this solution, the suppression factor for rates is exp $[-2 A]$. The contribution to $A$ from topology alone is $A_{t}$ $=8 \pi^{2} / e^{2}$. With use of the estimate of $e^{2}, 10^{-28}$ $\varsigma \exp \left[-2 A_{t}\right] \lessgtr 10^{-4}$. The remaining contributions to $\exp [-2 A]$ are hard to estimate as they depend sensitively on the cutoffs used for the meron size and the size of the zeros of the $\sigma$ field. Thus we regard our estimate for $\exp \left[-2 A_{t}\right]$ as a rough indication of the size of the suppression. Note that the error in identifying $A$ with $A_{t}$ overestimates $\exp [-2 A]$ since $A \gtrsim A_{t}$.

Summarizing, the salient results of this note are as follows:

(a) Long-lived and extended levels are expected to exist at the masses $1.9 \mathrm{GeV} \lessgtr M \lessgtr 5 \mathrm{GeV}$ and $1.8 \mathrm{GeV} s M+\mu \lesssim 5.6 \mathrm{GeV}$. Their baryon number and strangeness are expected to be $\gtrsim 6$ in magnitude. There are at least two levels at each of these masses; they are transformed into each other by charge conjugation. They correspond to $t= \pm 1$.

(b) Levels at $M$ or at $M+\mu$ are exotic, with fractional electric charge and "wrong" relation between baryon number and spin (in the sense explained before).

Anomalous nuclei with unexpectedly large lifetimes and cross sections $(\approx 10$ times normal cross sections) have been reported in the literature. ${ }^{15}$ It is conceivable that these are some of the levels we find. Although we do not know of a good reason why the cross sections of $t \neq 0$ states on normal matter should be enhanced, these states are poorly understood and such a enhancement cannot be ruled out.

A more detailed description of our work will be presented elsewhere.

We have benefitted greatly from discussions with Carl Rosenzweig. We also thank our other colleagues, especially C. G. Trahern, for advice and help.

This work was supported by the U. S. Department of Energy under Contracts No. DE-AC02-76 ER 03533 and No. DE-AS05-76 ER 03992.

${ }^{1}$ S. Weinberg, Phys. Rev. Lett. 18, 188 (1967), and Physica (Utrecht) 96A, 327 (1979).

${ }^{2}$ A. A. Slavnov, Nucl. Phys. B31, 301 (1971); S. Gerstein, R. Jackiw, B. W. Lee, and S. Weinberg, Phys. Rev. D 3 , 2486 (1971).

${ }^{3}$ T. H. R. Skyrme, Proc. Roy. Soc. London, Ser. A 260, 127 (1961), and Nucl. Phys. 31, 556 (1962).

${ }^{4}$ J. G. Williams, J. Math. Phys. (N.Y.) 11, 2611 (1970).

${ }^{5}$ N. K. Pak and H. Ch. Tze, Ann. Phys. (N.Y.) 117, 164 (1979).

${ }^{6}$ J. M. Gipson and H. Ch。Tze, Nucl. Phys. B183, 524 (1981).

${ }^{7}$ Yu.P. Rybakov and V. I. Sanyuk, to be published.

${ }^{8} \mathrm{~J}$. Goldstone and F. Wilczek, Phys. Rev. Lett. 47, 986 (1981), Eq. (6). [The boundary condition (3) differs from theirs.]

${ }^{9}$ R. Jackiw and C. Rebbi, Phys. Rev. D $\underline{13}, 3398$ (1976); R. Jackiw and J. R. Schrieffer, Nucl. Phys. B190, 253 (1981); R. Jackiw, Rev. Mod. Phys. 49, 681 $\overline{(1977)}$, and references therein.

${ }^{10}$ Compare A. Messiah, Quantum Mechanics (Wiley, New York, 1966), Vol. 2, p. 940.

${ }^{11}$ We may recall here that such states may be useful for fusion catalysis. See G. Zweig, Science 201, 973 (1978).

${ }^{12}$ B. R. Martin, D. Morgan, and G. Shaw, Pion-Pion Interactions in Particle Physics (Academic, New York, 1976), pp. 199, 262, 273, 376.

${ }^{13}$ Compare A. Actor, Rev. Mod. Phys. 51, 461 (1979). The meron $A_{\mu}$ may be written in terms of a chiral field $u$ as $\frac{1}{2} u^{+} \partial_{\mu} u$ which $u$ may be identified with the $u$ in the text.

${ }^{14}$ A. P. Balachandran, A. Stern, and C. G. Trahern, Phys. Rev. D 19, 2416 (1979).

${ }^{15}$ E. M. Friedlander, R. W. Gimpel, H. H. Heckman, Y. J. Karant, and B. Judek, Phys. Rev. Lett. 45, 1084 $(1980)$; H. B. Barber, P. S. Freier, and C. J. Waddington, Phys. Rev. Lett. $\underline{48}, 856$ (1982), and references therein. 